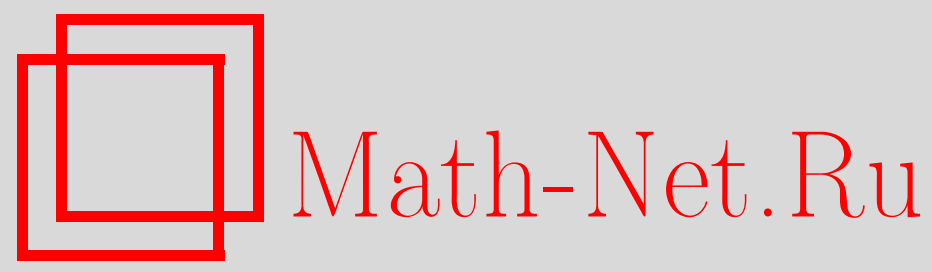

А. А. Шестаков, Исследование различных приближений при моделировании задач переноса теплового излучения, Матем. моделирование, 2020, том 32, номер 7, 77-97

DOI: https://doi.org/10.20948/mm-2020-07-05

Использование Общероссийского математического портала Math-Net.Ru подразумевает, что вы прочитали и согласны с пользовательским соглашением http://www.mathnet.ru/rus/agreement

Параметры загрузки:

IP : 35.173 .137 .237

26 апреля 2023 г., $17: 23: 25$

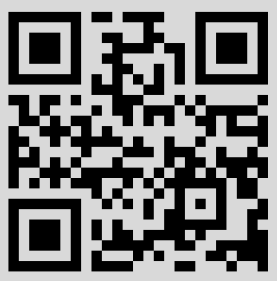




\section{ИССЛЕДОВАНИЕ РАЗЛИЧНЫХ ПРИБЛИЖЕНИЙ \\ ПРИ МОДЕЛИРОВАНИИ ЗАДАЧ ПЕРЕНОСА \\ ТЕПЛОВОГО ИЗЛУЧЕНИЯ}

(C) 2020 2. А.А. Шестаков

Российский Федеральный Ядерный Центр - Всероссийский НИИ технической физики им. акад. Е.И. Забабахина, Снежинск

a.a.shestakov2012@yandex.ru

DOI: $10.20948 / \mathrm{mm}-2020-07-05$

Целью данной работы является исследование и сравнение различных приближений системы уравнений переноса теплового излучения в оптически плотных и прозрачных средах. Для этого в оптически плотных средах используется асимптотический анализ, в оптически прозрачных средах - подход, позволяющий сводить решение диффузионных уравнений к решению кинетического уравнения. В результате проведенных исследований можно сказать, что в оптически плотных средах решения в рассмотренных приближениях стремятся к решению кинетического уравнения при увеличении оптической толщины. Это следует из асимптотического анализа. В оптически прозрачных средах совпадение с решением кинетического уравнения возможно только в приближениях квазипереноса и квазидиффузии.

Ключевые слова: перенос теплового излучения, приближения уравнения переноса.

\section{STUDY OF DIFFERENT APPROXIMATIONS FOR SOLVING HEAT TRANSFER}

\section{A.A. Shestakov}

Russian Federal Nuclear Center - Zababakhin All-Russian Research Institute of Technical Physics, Snezhinsk

The objective of this work is to study and compare different approximations to the system of equations which describe radiative heat transfer in optically thick and thin media. Asymptotic analysis is used for optically thick media. For optically thin media we use an approach that reduces diffusion equations to the kinetic one. Results of our study suggest that for optically thick media, solutions obtained with the approximations do converge to the solution of the kinetic equation as optical thickness increases. This follows from asymptotic analysis. For optically thin media, the convergence is only possible to quasitransport and quasi-diffusion solutions.

Keywords: radiative heat transfer, radiative heat transfer approximations. 


\section{Введение}

Одной из наиболее трудных задач теории переноса является решение спектральной системы уравнений переноса теплового излучения (СУПТИ), сложность которой определяется главным образом большой размерностью рассматриваемого пространства (7 независимых переменных в трехмерной геометрии). Из-за большого количества переменных решение уравнения переноса в трехмерной геометрии требует огромных вычислительных мощностей. В связи с этим для решения уравнения переноса применяют различные упрощающие приближения, сводящие задачу переноса излучения к более простой. К таким приближениям относятся групповое приближение [1], метод сферических гармоник [2], диффузионное приближение (Д) [3], приближение лучистой теплопроводности (ЛТП) [4] и другие.

Часть этих приближений не использует решение кинетического уравнения, а часть использует решение кинетического уравнения только для получения вспомогательных осредненных коэффициентов. Те приближения, которые не используют решение кинетического уравнения, дешевле, т.е. предпочтительнее по времени счета и объему занимаемой памяти ЭВМ, и дают в оптически плотных средах одинаковые с кинетической моделью решения. Поэтому они достаточно популярны и используются, в частности, в ядерных центрах США и России: Лос-Аламосской национальной лаборатории [5], Ливерморской национальной лаборатории [6], РФЯЦ-ВНИИЭФ $[7,8]$, РФЯЦ-ВНИИТФ $[9,10]$. Достоинством этих приближений, кроме удешевления расчетов по сравнению с кинетической постановкой, является сохранение симметрии в сферически-симметричных задачах. Это снимает вопрос о влиянии лучевого эффекта в кинетической постановке, который, например, может влиять на форму газа в задачах лазерного термоядерного синтеза (ЛТС), что приводит к нефизическому распределению веществ при больших значениях объемного сжатия, типичных для мишеней ЛТС. Однако они имеют ограничения по области применения, поэтому их используют в основном в оптически плотных веществах.

Те приближения, которые используют решение кинетического уравнения, дороже и сложнее в реализации, но не имеют ограничений по области применения. Исторически первым таким приближением в России было квазидиффузионное приближение (КД), предложенное Гольдиным в 1964 году [11], и в дальнейшем использованное многими специалистами (см., например, $[12,13])$. В последнее время для таких приближений используется термин HOLO-алгоритмы [14], в которых решение кинетического уравнения ищется как на уровне решения уравнений высокой размерности (HO - high order), так и на уровне уравнений низкой размерности (LO - low order). 
В настоящее время появилось еще одно приближение [15-17], использующее решение кинетического уравнения для получения вспомогательных коэффициентов. Это приближение получило название квазипереноса (КП). В связи с этим возникла необходимость в изучении свойств нового приближения КП и сравнении его с ранее используемыми автором приближениями: Д [18], $\mathrm{P}_{1}[19], \mathrm{P}_{1 / 3}$ [20] и КД [21-24].

При построении КД и КП приближений решение СУПТИ разбивается на два этапа. На первом этапе в этих приближениях решается кинетическое уравнение для получения вспомогательных осредненных коэффициентов. На втором этапе решается квазидиффузионное или диффузионное уравнения, размерности которых на 2 переменные меньше, для получения основных величин: температуры, плотности и потока излучения.

В отличие от двухэтапных итерационных методов: LM [25], DSA [26], RDSA [27], P1SA [28,29], где требуется согласованность схем на этапах, а второй этап используется для ускорения итераций, в КД и КП приближениях не требуется согласованность схем на этапах, а вся тяжесть итерационного процесса переносится на второй этап, который считается с поправочными коэффициентами, при этом кинетическое уравнение решается один раз на временном шаге.

Основные вопросы, которые рассматриваются в статье, - в каких приближениях и при каких условиях можно получить решения, совпадающие с решением кинетического уравнения, и если - нет, то что нужно сделать, чтобы максимально сблизить эти решения. Найти области применения рассмотренных приближений. Аналогичные исследования были сделаны в [30].

Исследования проведены в оптически плотных и прозрачных средах для сферически-симметричной и плоской геометрий.

\section{1. Постановка задачи}

Система уравнений, описывающая распространение излучения в неподвижной среде, состоит из спектрального уравнения переноса (УП) в кинетической постановке, описывающего перенос, поглощение и рассеяние теплового излучения, и уравнения энергии, характеризующего изменение температуры вещества за счет поглощения и испускания фотонов. Система уравнений в сферически-симметричном и плоском случаях без учета движения среды для изотропного рассеяния выглядит следующим образом [31]:

$$
\left\{\begin{array}{l}
\frac{1}{c} \frac{\partial J_{v}}{\partial t}+\boldsymbol{\Omega} \nabla J_{v}+\alpha_{v} J_{v}=\frac{1}{2} \alpha_{c v} B_{v}+\frac{1}{2} \alpha_{s v} U_{v}, \\
\frac{\partial E}{\partial t}=\int_{0}^{\infty} \alpha_{c v}\left(U_{v}-B_{v}\right) d v+\frac{\partial Q}{\partial t} .
\end{array}\right.
$$


Здесь $J_{v}(r, \mu, v, t)$ - спектральная интенсивность излучения, $T(r, t)$ - температура среды, $\alpha_{v}=\alpha_{c v}+\alpha_{s v}-$ коэффициент ослабления, $\alpha_{c v}-$ коэффициент поглощения, $\alpha_{s v}-$ коэффициент рассеяния, $E(T)$ - удельная внутренняя энергия вещества, $B_{v}(v, T)$ - плотность равновесного излучения, умноженная на $c, U_{v}=\int_{-1}^{1} J_{v} d \mu-$ спектральная плотность энергии излучения, умноженная на $c, c$ - скорость света, $r$ - пространственная координата, $t-$ время, $v$ - частота фотонов, $\mu$ - косинус угла между направлением полёта фотона $\boldsymbol{\Omega}$ и осью r, $Q$ - источник, $\boldsymbol{\Omega} \nabla J_{v}=\frac{\mu}{r^{\eta}} \frac{\partial}{\partial r}\left(r^{\eta} J_{v}\right)+\frac{\eta}{2 r} \frac{\partial}{\partial \mu}\left(\left(1-\mu^{2}\right) J_{v}\right)$, $\eta$ - параметр геометрии (0 - плоская, 2 - сферически-симметричная).

Система переноса излучения в КД приближении на втором этапе имеет вид

$$
\begin{aligned}
& \frac{1}{c} \frac{\partial U_{v}}{\partial t}+\frac{1}{r^{\eta}} \frac{\partial\left(r^{\eta} S_{v}\right)}{\partial r}+\alpha_{c v} U_{v}=\alpha_{c v} B_{v}, \\
& \frac{1}{c} \frac{\partial S_{v}}{\partial t}+\frac{\partial D_{v} U_{v}}{\partial r}+\frac{\eta}{2} \frac{3 D_{v}-1}{r} U_{v}+\alpha_{v} S_{v}=0
\end{aligned}
$$

где $S_{v}=\int_{-1}^{1} \mu J_{v} d \mu$ - спектральный поток излучения, $D_{v}=\left(\int_{-1}^{1} J_{v} d \mu\right)^{-1} \times$ $\times \int_{-1}^{1} \mu^{2} J_{v} d \mu-$ коэффициент КД.

Система переноса излучения в КП приближении на втором этапе имеет вид

$$
\begin{aligned}
& \frac{1}{c} \frac{\partial U_{v}}{\partial t}+\frac{1}{r^{\eta}} \frac{\partial\left(r^{\eta} S_{v}\right)}{\partial r}+\alpha_{c v} U_{v}=\alpha_{c v} B_{v}, \\
& \frac{m_{v}}{3} \frac{\partial U_{v}}{\partial r}+\alpha_{v} S_{v}=0
\end{aligned}
$$

где $m_{v}=-\left(\frac{1}{3 \alpha_{v}} \frac{\partial}{\partial r} \int_{-1}^{1} J_{v} d \mu\right)^{-1} \int_{-1}^{1} \mu J_{v} d \mu-$ коэффициент КП.

Система переноса излучения в Д, $\mathrm{P}_{1}$ и $\mathrm{P}_{1 / 3}$ приближениях имеет вид

$$
\begin{aligned}
& \frac{1}{c} \frac{\partial U_{v}}{\partial t}+\frac{1}{r^{\eta}} \frac{\partial\left(r^{\eta} S_{v}\right)}{\partial r}+\alpha_{c v} U_{v}=\alpha_{c v} B_{v}, \\
& \underline{\beta} \frac{\partial S_{v}}{\partial t}+\frac{1}{3} \frac{\partial U_{v}}{\partial r}+\alpha_{v} S_{v}=0
\end{aligned}
$$

где $\beta=0$ - диффузионное приближение, $\beta=1-\mathrm{P}_{1}$ приближение, $\beta=1 / 3-\mathrm{P}_{1 / 3}$ приближение. 
Система переноса излучения в ЛТП приближении имеет вид

$$
\begin{aligned}
& \frac{\partial \varepsilon}{\partial t}+\frac{1}{r^{\eta}} \frac{\partial\left(r^{\eta} S\right)}{\partial r}=\frac{\partial Q}{\partial t}, \\
& S=-\chi \frac{\partial}{\partial r} T,
\end{aligned}
$$

где $\varepsilon=E+B / c$ - тепловая энергия с учетом излучения, $S$ - поток, $\chi=\frac{4 c \sigma l}{3} T^{3}=5480 l T^{3}-$ коэффициент теплопроводности, $l-$ пробег, $B=c \sigma T^{4}$, $\sigma=4 c^{-1} \sigma_{S B}, \sigma_{S B}-$ постоянная Стефана-Больцмана.

\section{2. Сравнение решений уравнения переноса в различных приближениях в оптически плотной среде}

Совпадение решений СУПТИ в различных приближениях с кинетическим решением в оптически плотной среде дает асимптотический анализ этих уравнений, предложенный в [32]. В этой работе исследуется диффузионный предел СУПТИ, суть которого заключается в том, что в оптически плотных средах решение кинетического уравнения раскладывается в ряд, старшие члены которого являются решением диффузионного уравнения. При увеличении оптической толщины решение ряда стремится к решению диффузионного уравнения.

$\mathrm{B}$ данной работе асимптотический анализ проведен для $\mathrm{P}_{1}, \mathrm{P}_{1 / 3}, \mathrm{KД}$, КП приближений. Для исследования диффузионного предела уравнения переноса в различных приближениях рассматривается среда, где рассеяние преобладает над поглощением, т.е. $\alpha_{s v} \gg \alpha_{c v}$, а изменения по времени и пространству происходят очень медленно. Вводим новые переменные времени и пространства, необходимые для асимптотического анализа $x=\varepsilon r, t^{\prime}=\varepsilon^{2} t$, где $\varepsilon-$ некоторый малый параметр (обычно отношение среднего пути свободного пробега к размеру рассматриваемой системы).

В КД и КП приближениях СУПТИ решается в два этапа. На первом этапе уравнение переноса в новых переменных приобретает вид

$$
\frac{\varepsilon^{2}}{c} \frac{\partial J_{v}}{\partial t^{\prime}}+\frac{\mu \varepsilon}{x^{\eta}} \frac{\partial}{\partial x}\left(x^{\eta} J_{v}\right)+\frac{\eta \varepsilon}{2 x} \frac{\partial}{\partial \mu}\left(\left(1-\mu^{2}\right) J_{v}\right)+\alpha_{v} J_{v}=\frac{1}{2} \alpha_{c v} B_{v}+\frac{1}{2} \alpha_{s v} U_{v} .
$$

На втором этапе уравнения в КД приближении (2) приобретают вид

$$
\begin{aligned}
& \frac{\varepsilon^{2}}{c} \frac{\partial U_{v}}{\partial t^{\prime}}+\frac{\varepsilon}{x^{\eta}} \frac{\partial\left(x^{\eta} S_{v}\right)}{\partial x}+\alpha_{c v} U_{v}=\alpha_{c v} B_{v}, \\
& \frac{\varepsilon^{2}}{c} \frac{\partial S_{v}}{\partial t^{\prime}}+\varepsilon \frac{\partial\left(D_{v} U_{v}\right)}{\partial x}+\frac{\eta \varepsilon}{2} \frac{3 D_{v}-1}{x} U_{v}+\alpha_{v} S_{v}=0 .
\end{aligned}
$$


В КП приближении уравнения (3) приобретают вид

$$
\begin{aligned}
& \frac{\varepsilon^{2}}{c} \frac{\partial U_{v}}{\partial t^{\prime}}+\frac{\varepsilon}{x^{\eta}} \frac{\partial\left(x^{\eta} S_{v}\right)}{\partial x}+\alpha_{c v} U_{v}=\alpha_{c v} B_{v}, \\
& \frac{m_{v} \varepsilon}{3} \frac{\partial U_{v}}{\partial x}+\alpha_{v} S_{v}=0
\end{aligned}
$$

где $m_{v}=-\left(\frac{\varepsilon}{3 \alpha_{v}} \frac{\partial}{\partial x} \int_{-1}^{1} J_{v} d \mu\right)^{-1} \int_{-1}^{1} \mu J_{v} d \mu$.

Система переноса излучения в $\mathrm{P}_{1}$ и $\mathrm{P}_{1 / 3}$ приближениях (4) в новых переменных приобретает вид

$$
\begin{aligned}
& \frac{\varepsilon^{2}}{c} \frac{\partial U_{v}}{\partial t^{\prime}}+\frac{\varepsilon}{x^{\eta}} \frac{\partial\left(x^{\eta} S_{v}\right)}{\partial x}+\alpha_{c v} U_{v}=\alpha_{c v} B_{v}, \\
& \frac{\beta \varepsilon^{2}}{c} \frac{\partial S_{v}}{\partial t^{\prime}}+\frac{\varepsilon}{3} \frac{\partial U_{v}}{\partial x}+\alpha_{v} S_{v}=0 .
\end{aligned}
$$

Уравнение энергии в новых переменных приобретает вид:

$$
\varepsilon^{2} \frac{\partial E}{\partial t^{\prime}}=\int_{0}^{\infty} \alpha_{c v}\left(U_{v}-B_{v}\right) d v+\varepsilon^{2} \frac{\partial Q}{\partial t^{\prime}} .
$$

При $\varepsilon=1$ уравнения (6)-(10) в новых переменных совпадают с исходными уравнениями (1)-(4).

Рассмотрим асимптотическое разложение основных величин в бесконечные ряды:

$$
\begin{array}{ll}
J_{v}\left(t^{\prime}, x, \mu\right)=\sum_{j=0}^{\infty} \varepsilon^{j} J_{v}^{(j)}\left(t^{\prime}, x, \mu\right), & U_{v}\left(t^{\prime}, x\right)=\sum_{j=0}^{\infty} \varepsilon^{j} U_{v}^{(j)}\left(t^{\prime}, x\right), \\
B_{v}\left(t^{\prime}, x\right)=\sum_{j=0}^{\infty} \varepsilon^{j} B_{v}^{(j)}\left(t^{\prime}, x\right), & S_{v}\left(t^{\prime}, x\right)=\sum_{j=0}^{\infty} \varepsilon^{j} S_{v}^{(j)}\left(t^{\prime}, x\right),
\end{array}
$$

где для коэффициентов разложения выполняются следующие интегральные соотношения: $U_{v}^{(j)}=\int_{-1}^{1} J_{v}^{(j)} d \mu, S_{v}^{(j)}=\int_{-1}^{1} \mu J_{v}^{(j)} d \mu$.

Покажем, что уравнения (6)-(10) имеют диффузионный предел при $\varepsilon \rightarrow 0$ для старших членов разложения.

Подставляя вышеперечисленные выражения в системы (6)-(10) и приравнивая члены разложения с одинаковой степенью $\varepsilon$, получаем уравнения для нахождения всех членов разложения в ряды.

Для кинетического уравнения (6) с уравнением энергии (10) при $\varepsilon^{0}$ получаем 


$$
\left\{\begin{array}{l}
\alpha_{v} J_{v}^{(0)}=\frac{1}{2} \alpha_{c v} B_{v}^{(0)}+\frac{1}{2} \alpha_{s v} U_{v}^{(0)}, \\
\int_{0}^{\infty} \alpha_{c v}\left(U_{v}^{(0)}-B_{v}^{(0)}\right) d v=0 .
\end{array}\right.
$$

После интегрирования первого уравнения системы (11) по $\mu$ получаем для старших членов разложения плотности излучения планковское распределение: $U_{v}^{(0)}=B_{v}^{(0)}$. Подставляя это выражение в систему (11), получаем планковское распределение для интенсивности:

$$
J_{v}^{(0)}=\frac{1}{2 \alpha_{v}}\left(\alpha_{c v} B_{v}^{(0)}+\alpha_{s v} U_{v}^{(0)}\right)=\frac{1}{2} B_{v}^{(0)} .
$$

После интегрирования первого уравнения системы (11) по $\mu$ с весом $\mu$, получаем условие локального термодинамического равновесия (ЛТР): $S_{v}^{(0)}=0$. Приравнивая члены разложения при $\varepsilon^{1}$ после интегрирования уравнения (6) по $\mu$, получаем стационарное диффузионное уравнение:

$$
\frac{\partial\left(x^{\eta} S_{v}^{(0)}\right)}{x^{\eta} \partial x}+\alpha_{c v} U_{v}^{(1)}=\alpha_{c v} B_{v}^{(1)},
$$

которое с учетом полученного выше значения $S_{v}^{(0)}=0$ дает планковское распределение для второго члена разложения: $U_{v}^{(1)}=B_{v}^{(1)}$.

Для $\varepsilon^{1}$ после интегрирования уравнения (6) по $\mu$ с весом $\mu$ получаем уравнение:

$$
\frac{\partial}{\partial x}\left(D_{v}^{(0)} U_{v}^{(0)}\right)+\frac{\eta}{2} \frac{3 D_{v}^{(0)}-1}{x} U_{v}^{(0)}+\alpha_{v} S_{v}^{(1)}=0
$$

где $D_{v}^{(0)}=\int_{-1}^{1} \mu^{2} J_{v}^{(0)} d \mu / U_{v}^{(0)}=\frac{1}{2} \int_{-1}^{1} \mu^{2} B_{v}^{(0)} d \mu / B_{v}^{(0)}=\frac{1}{2} \int_{-1}^{1} \mu^{2} d \mu=\frac{1}{3}$.

При $D_{v}^{(0)}=1 / 3$ это уравнение совпадает с законом Фика для диффузионного потока $S_{v}^{(1)}=-\frac{1}{3 \alpha_{v}} \frac{\partial U_{v}^{(0)}}{\partial x}$ и после интегрирования по частоте с учетом $U_{v}^{(0)}=B_{v}^{(0)}$ совпадает со вторым уравнением системы ЛТП (5), которое является законом Фурье для теплопроводного потока:

$$
S^{(1)}=-\frac{1}{3 \alpha_{R}} \frac{\partial B^{(0)}}{\partial x}=-\frac{4 c \sigma T^{3}}{3 \alpha_{R}} \frac{\partial T}{\partial x},
$$


где $l_{R}=\frac{1}{\alpha_{R}}=\frac{\int_{0}^{\infty} \frac{1}{\alpha_{v}} \frac{\partial B_{v}^{(0)}}{\partial x} d v}{\int_{0}^{\infty} \frac{\partial B_{v}^{(0)}}{\partial x} d v}-$ росселандов пробег, $B^{(0)}=\int_{0}^{\infty} B_{v}^{(0)} d v=c \sigma T^{4}$.

Подставляя полученные выражения в УП, получаем при $\varepsilon^{1}$ :

$$
\frac{\mu}{x^{\eta}} \frac{\partial}{\partial x}\left(x^{\eta} J_{v}^{(0)}\right)+\frac{\eta}{2 x} \frac{\partial}{\partial \mu}\left(\left(1-\mu^{2}\right) J_{v}^{(0)}\right)+\alpha_{v} J_{v}^{(1)}=\frac{1}{2} \alpha_{c v} B_{v}^{(1)}+\frac{1}{2} \alpha_{s v} B_{v}^{(1)} .
$$

Учитывая $J_{v}^{(0)}=\frac{1}{2} B_{v}^{(0)}$, получаем $J_{v}^{(1)}=\frac{1}{2} B_{v}^{(1)}-\frac{\mu}{2 \alpha_{v}} \frac{\partial B_{v}^{(0)}}{\partial x}$.

Приравнивая члены разложения при $\varepsilon^{2}$ после интегрирования уравнения (6) по н, получаем уравнение:

$$
\frac{1}{c} \frac{\partial U_{v}^{(0)}}{\partial t^{\prime}}+\frac{\partial\left(x^{\eta} S_{v}^{(1)}\right)}{x^{\eta} \partial x}+\alpha_{c v} U_{v}^{(2)}=\alpha_{c v} B_{v}^{(2)} .
$$

После суммирования с уравнением энергии $\frac{\partial E}{\partial t^{\prime}}=\int_{0}^{\infty} \alpha_{c v}\left(U_{v}^{(2)}-B_{v}^{(2)}\right) d v+\frac{\partial Q}{\partial t^{\prime}}$ получаем уравнение теплового баланса, совпадающее с первым уравнением системы ЛТП (5): $\frac{\partial}{\partial t^{\prime}}\left(E+\frac{1}{c} B^{(0)}\right)+\frac{\partial\left(x^{\eta} S^{(1)}\right)}{x^{\eta} \partial x}=\frac{\partial Q}{\partial t^{\prime}}$.

Для $\varepsilon^{2}$ после интегрирования уравнения (6) по $\mu$ с весом $\mu$ получаем уравнение:

$$
\frac{1}{c} \frac{\partial S_{v}^{(0)}}{\partial t^{\prime}}+\frac{\partial}{\partial x}\left(D_{v}^{(1)} U_{v}^{(1)}\right)+\frac{\eta}{2} \frac{3 D_{v}^{(1)}-1}{x} U_{v}^{(1)}+\alpha_{v} S_{v}^{(2)}=0,
$$

где

$$
\begin{aligned}
& D_{v}^{(1)}=\int_{-1}^{1} \mu^{2} J_{v}^{(1)} d \mu / U_{v}^{(1)}=\frac{1}{2 B_{v}^{(1)}} \int_{-1}^{1} \mu^{2}\left\{B_{v}^{(1)}-\frac{\mu}{\alpha_{v}}\left[\frac{1}{x^{\eta}} \frac{\partial}{\partial x}\left(x^{\eta} B_{v}^{(0)}\right)-\frac{\eta}{x} B_{v}^{(0)}\right]\right\} d \mu= \\
& =\frac{1}{2} \int_{-1}^{1} \mu^{2} d \mu=\frac{1}{3} .
\end{aligned}
$$

Это уравнение совпадает с законом Фика для диффузионного потока $S_{v}^{(2)}=$ $=-\frac{1}{3 \alpha_{v}} \frac{\partial B_{v}^{(1)}}{\partial x}$ и после интегрирования по частоте совпадает со вторым уравнением системы ЛТП.

Подставляя полученные выражения в УП, получаем при $\varepsilon^{2}$ : 


$$
\frac{1}{c} \frac{\partial J_{v}^{(0)}}{\partial t^{\prime}}+\frac{\mu}{x^{\eta}} \frac{\partial}{\partial x}\left(x^{\eta} J_{v}^{(1)}\right)+\frac{\eta}{2 x} \frac{\partial}{\partial \mu}\left(\left(1-\mu^{2}\right) J_{v}^{(1)}\right)+\alpha_{v} J_{v}^{(2)}=\frac{1}{2} \alpha_{c v} B_{v}^{(2)}+\frac{1}{2} \alpha_{s v} U_{v}^{(2)} \text {. }
$$

Учитывая

$$
\begin{aligned}
& J_{v}^{(0)}=\frac{1}{2} B_{v}^{(0)}, \quad J_{v}^{(1)}=\frac{1}{2} B_{v}^{(1)}-\frac{\mu}{2 \alpha_{v}}\left[\frac{1}{x^{\eta}} \frac{\partial}{\partial x}\left(x^{\eta} B_{v}^{(0)}\right)-\frac{\eta}{x} B_{v}^{(0)}\right], \\
& U_{v}^{(2)}=B_{v}^{(2)}-\frac{1}{\alpha_{c v}}\left[\frac{1}{c} \frac{\partial B_{v}^{(0)}}{\partial t^{\prime}}-\frac{\partial}{x^{\eta} \partial x}\left(\frac{x^{\eta}}{3 \alpha_{v}} \frac{\partial B_{v}^{(0)}}{\partial x}\right)\right],
\end{aligned}
$$

получаем уравнение для нахождения $J_{v}^{(2)}$ :

$$
\begin{aligned}
& J_{v}^{(2)}=\frac{1}{2} B_{v}^{(2)}-\frac{1}{2} \frac{\alpha_{s v}}{\alpha_{v} \alpha_{c v}}\left[\frac{1}{c} \frac{\partial B_{v}^{(0)}}{\partial t^{\prime}}-\frac{\partial}{x^{\eta} \partial x}\left(\frac{x^{\eta}}{3 \alpha_{v}} \frac{\partial B_{v}^{(0)}}{\partial x}\right)\right]-\frac{1}{2 c \alpha_{v}} \frac{\partial B_{v}^{(0)}}{\partial t^{\prime}}- \\
& -\frac{\mu}{x^{\eta} \alpha_{v}} \frac{\partial}{\partial x}\left(x^{\eta}\left\{\frac{1}{2} B_{v}^{(1)}-\frac{\mu}{2 \alpha_{v}} \frac{\partial B_{v}^{(0)}}{\partial x}\right\}\right)-\frac{\eta}{2 x \alpha_{v}} \frac{\partial}{\partial \mu}\left(\left(1-\mu^{2}\right)\left\{\frac{1}{2} B_{v}^{(1)}-\frac{\mu}{2 \alpha_{v}} \frac{\partial B_{v}^{(0)}}{\partial x}\right\}\right) .
\end{aligned}
$$

Собирая все члены разложения в ряды, получаем окончательный вид решения:

$$
\begin{aligned}
& J_{v}=\frac{1}{2}\left(B_{v}^{(0)}+\varepsilon B_{v}^{(1)}\right)-\frac{\mu \varepsilon}{2 \alpha_{v}} \frac{\partial B_{v}^{(0)}}{\partial x}+O\left(\varepsilon^{2}\right)=\frac{1}{2} B_{v}+O(\varepsilon), \\
& U_{v}=B_{v}^{(0)}+\varepsilon B_{v}^{(1)}+\varepsilon^{2}\left\{B_{v}^{(2)}-\frac{1}{\alpha_{c v}}\left[\frac{1}{c} \frac{\partial B_{v}^{(0)}}{\partial t^{\prime}}-\frac{\partial}{x^{\eta} \partial x}\left(\frac{x^{\eta}}{3 \alpha_{v}} \frac{\partial B_{v}^{(0)}}{\partial x}\right)\right]\right\}+ \\
& +O\left(\varepsilon^{3}\right)=B_{v}+O\left(\varepsilon^{2}\right), \\
& S_{v}=-\frac{1}{3 \alpha_{v}}\left\{\varepsilon \frac{\partial B_{v}^{(0)}}{\partial x}+\varepsilon^{2} \frac{\partial B_{v}^{(1)}}{\partial x}\right\}+O\left(\varepsilon^{2}\right)=-\frac{\varepsilon}{3 \alpha_{v}} \frac{\partial B_{v}}{\partial x}+O\left(\varepsilon^{2}\right), \\
& B_{v}=B_{v}^{(0)}+\varepsilon B_{v}^{(1)}+\varepsilon^{2} B_{v}^{(2)}+O\left(\varepsilon^{3}\right) .
\end{aligned}
$$

Из этой системы видно, что при асимптотическом разложении из кинетического уравнения для старших членов выполняется диффузионный предел.

Перейдем к рассмотрению уравнений (9) в $\mathrm{P}_{1}$ и $\mathrm{P}_{1 / 3}$ приближениях.

При $\varepsilon^{0}$ получаем $U_{v}^{(0)}=B_{v}^{(0)}, S_{v}^{(0)}=0$. При $\varepsilon^{1}$ получаем $U_{v}^{(1)}=B_{v}^{(1)}$, $S_{v}^{(1)}=-\frac{1}{3 \alpha_{v}} \frac{\partial B_{v}^{(0)}}{\partial x}$, откуда следует диффузионный предел. При $\varepsilon^{2}$ получаем следующие уравнения для $U_{v}^{(2)}$ и $S_{v}^{(2)}$ : 
$\frac{1}{c} \frac{\partial U_{v}^{(0)}}{\partial t^{\prime}}+\frac{\partial\left(x^{\eta} S_{v}^{(1)}\right)}{x^{\eta} \partial x}+\alpha_{c v} U_{v}^{(2)}=\alpha_{c v} B_{v}^{(2)}, \quad \frac{\beta}{c} \frac{\partial S_{v}^{(0)}}{\partial t^{\prime}}+\frac{1}{3} \frac{\partial U_{v}^{(1)}}{\partial x}+\alpha_{v} S_{v}^{(2)}=0$.

Отсюда следует диффузионный предел при $\varepsilon^{2}$ :

$U_{v}^{(2)}=B_{v}^{(2)}-\frac{1}{\alpha_{c v}}\left[\frac{1}{c} \frac{\partial B_{v}^{(0)}}{\partial t^{\prime}}-\frac{\partial}{x^{\eta} \partial x}\left(\frac{x^{\eta}}{3 \alpha_{v}} \frac{\partial B_{v}^{(0)}}{\partial x}\right)\right], \quad S_{v}^{(2)}=-\frac{1}{3 \alpha_{v}} \frac{\partial B_{v}^{(1)}}{\partial x}$.

Рассмотрим уравнения второго этапа (7), (8) в КД и КП приближениях. Коэффициент $D_{v}$ непосредственно в ряд не раскладывается, а рассматривается выражение $D_{v}^{(j)}=\frac{\int_{-1}^{1} \mu^{2} J_{v}^{(j)} d \mu}{\int_{-1}^{1} J_{v}^{(j)} d \mu}$. В системе (7) член $D_{v} U_{v}$ можно рассматривать в виде следующего разложения

$$
\begin{aligned}
& D_{v} U_{v}=\int_{-1}^{1} \mu^{2} J_{v} d \mu=\int_{-1}^{1} \mu^{2}\left(\sum_{j=0}^{\infty} \varepsilon^{j} J_{v}^{(j)}\right) d \mu=\sum_{j=0}^{\infty} \varepsilon^{j} \int_{-1}^{1} \mu^{2} J_{v}^{(j)} d \mu= \\
& =\sum_{j=0}^{\infty} \varepsilon^{j} D_{v}^{(j)} \int_{-1}^{1} J_{v}^{(j)} d \mu=\sum_{j=0}^{\infty} \varepsilon^{j} D_{v}^{(j)} U_{v}^{(j)},
\end{aligned}
$$

где $J_{v}^{(j)}$ вычисляется из кинетического уравнения на первом этапе и совпадает с разложением (12). Подставляя это разложение в систему (7), получаем при $\varepsilon^{0}: U_{v}^{(0)}=B_{v}^{(0)}, S_{v}^{(0)}=0, D_{v}^{(0)}=1 / 3$. При $\varepsilon^{1}$ получаем $U_{v}^{(1)}=B_{v}^{(1)}$, $S_{v}^{(1)}=-\frac{1}{3 \alpha_{v}} \frac{\partial B_{v}^{(0)}}{\partial x}, D_{v}^{(1)}=1 / 3$. При $\varepsilon^{2}$ получаем аналогичные (16) выражения для $U_{v}^{(2)}$ и $S_{v}^{(2)}$, но более сложные выражения для $J_{v}^{(2)}$ и $D_{v}^{(2)}$, поэтому в данной работе они не приводятся.

В КП приближении коэффициент $m$ рассматривается в виде

$$
m_{v}=-\left(\frac{\varepsilon}{3 \alpha_{v}} \frac{\partial}{\partial x} \int_{-1}^{1} J_{v} d \mu\right)^{-1} \int_{-1}^{1} \mu J_{v} d \mu=\frac{-\frac{1}{3 \alpha_{v}}\left(\varepsilon \frac{\partial B_{v}^{(0)}}{\partial x}+\varepsilon^{2} \frac{\partial B_{v}^{(1)}}{\partial x}\right)+O\left(\varepsilon^{2}\right)}{-\frac{\varepsilon}{3 \alpha_{v}} \frac{\partial}{\partial x}\left(B_{v}^{0}+\varepsilon B_{v}^{(1)}+O\left(\varepsilon^{2}\right)\right)}=1+O(\varepsilon) .
$$

При разложении в ряд уравнение для потока в системе (8) умножается на знаменатель коэффициента КП и рассматривается в виде

$$
\frac{\varepsilon}{3} \int_{-1}^{1} \mu J_{v} d \mu \frac{\partial U_{v}}{\partial x}-\alpha_{v} S_{v} \frac{\varepsilon}{3 \alpha_{v}} \frac{\partial}{\partial x} \int_{-1}^{1} J_{v} d \mu=0
$$


где $J_{v}$ вычисляется из кинетического уравнения на первом этапе. Используя разложение (12) для интенсивности на первом этапе, из уравнения (17) получаем уравнение для нахождения потока $S_{v}=\sum_{j=0}^{\infty} \varepsilon^{j} S_{v}^{(j)}$ на втором этапе КП:

$$
\begin{aligned}
& \varepsilon\left\{-\frac{1}{3 \alpha_{v}}\left\{\varepsilon \frac{\partial B_{v}^{(0)}}{\partial x}+\varepsilon^{2} \frac{\partial B_{v}^{(1)}}{\partial x}\right\}+O\left(\varepsilon^{2}\right)\right\} \frac{\partial}{\partial x} \sum_{j=0}^{\infty} \varepsilon^{j} U_{v}^{(j)}= \\
& =\varepsilon \frac{\partial}{\partial x}\left(B_{v}^{(0)}+\varepsilon B_{v}^{(1)}+\varepsilon^{2}\left\{B_{v}^{(2)}-\frac{1}{\alpha_{c v}}\left[\frac{1}{c} \frac{\partial B_{v}^{(0)}}{\partial t^{\prime}}-\right.\right.\right. \\
& \left.\left.\left.-\frac{\partial}{x^{\eta} \partial x}\left(\frac{x^{\eta}}{3 \alpha_{v}} \frac{\partial B_{v}^{(0)}}{\partial x}\right)\right]\right\}+O\left(\varepsilon^{3}\right)\right) \sum_{j=0}^{\infty} \varepsilon^{j} S_{v}^{(j)} .
\end{aligned}
$$

При $\varepsilon^{0}$ получаем $U_{v}^{(0)}=B_{v}^{(0)}, S_{v}^{(0)}=0$. При $\varepsilon^{1}$ получаем $U_{v}^{(1)}=B_{v}^{(1)}, S_{v}^{(1)}=$ $=-\frac{1}{3 \alpha_{v}} \frac{\partial B_{v}^{(0)}}{\partial x}$. При $\varepsilon^{2}$ получаем аналогичные (16) выражения для $U_{v}^{(2)}$ и $S_{v}^{(2)}$.

Видно, что для старших членов разложения при $\varepsilon^{1}$ и $\varepsilon^{2}$ в КД и КП приближениях выполняется диффузионный предел.

Следует заметить, что при разностной аппроксимации уравнения переноса в различных приближениях асимптотический диффузионный предел выполняется не всегда, поэтому для каждой конкретной численной реализации его надо исследовать отдельно. В [33] проведен анализ системы Рn уравнений для схемы Римана и показано, что для этой схемы асимптотический диффузионный предел не выполняется. В [34] проведен анализ системы $\mathrm{P}_{1}$ уравнений для схемы Ромб и показано, что асимптотический диффузионный предел выполняется в схеме Ромб.

Перейдем к исследованию приближений КД, КП, Д, $\mathrm{P}_{1}$ и $\mathrm{P}_{1 / 3}$ в случае оптически прозрачных сред.

\section{3. Сравнение решений стационарного уравнения переноса в различных приближениях в оптически прозрачной среде}

Для сравнения решений УП в оптически прозрачной среде вначале рассмотрим вакуум, который является предельным случаем оптически прозрачных сред при $\alpha \rightarrow 0$. Исследуем решение СУПТИ (1) в сером приближении для стационарных задач. В вакууме стационарное уравнение переноса излучения для сферически-симметричной и плоской геометрий имеет вид

$$
\boldsymbol{\Omega} \nabla J=\frac{\mu}{r^{\eta}} \frac{\partial}{\partial r}\left(r^{\eta} J\right)+\frac{\eta}{2 r} \frac{\partial}{\partial \mu}\left(\left(1-\mu^{2}\right) J\right)=0 .
$$


После интегрирования по всем направлениям из (19) получаем обыкновенное дифференциальное уравнение (ОДУ): $\left(\partial r^{\eta} S\right) / \partial r=0$, из которого следует точное решение для потока

$r^{\eta} S=$ const.

Уравнение для нахождения плотности излучения из соотношения (19) получить не удается, поэтому точное решение для плотности можно получить только в случае, если известно решение для интенсивности

$$
U=\int_{-1}^{1} J d \mu .
$$

В вакууме стационарная сферически-симметричная система переноса излучения в Д, $\mathrm{P}_{1}$ и $\mathrm{P}_{1 / 3}$ приближениях имеет одинаковый вид:

$$
\begin{aligned}
& \frac{1}{r^{\eta}} \frac{\partial r^{\eta} S}{\partial r}=0 \\
& \frac{1}{3} \frac{\partial U}{\partial r}=0 .
\end{aligned}
$$

Из решения этой системы следует точное решение для потока $r^{\eta} S=$ const и плотности $U=$ const .

Следует заметить, что решение в кинетическом приближении не является решением системы в Д, $\mathrm{P}_{1}$ и $\mathrm{P}_{1 / 3}$ приближениях, т.к. может не удовлетворять второму уравнению для производной по $\mathrm{U}: \frac{\partial}{\partial r} \int_{-1}^{1} J d \mu=0$. Из кинетического уравнения можно получить дополнительные уравнения, проинтегрировав УП с некоторыми весами в пространстве направлений. При интегрировании стационарного уравнения переноса по всем направлениям полета фотонов с весом $\mu$ получаем $\frac{1}{r^{\eta}} \frac{\partial}{\partial r} \int_{-1}^{1} \mu^{2} r^{\eta} J d \mu+\frac{\eta}{2 r} \int_{-1}^{1}\left(\mu^{2}-1\right) J d \mu=0$.

Это уравнение можно привести к виду относительно плотности $\frac{1}{r^{\eta}} \frac{\partial}{\partial r}\left(r^{\eta} D U\right)+\frac{\eta}{2 r}(D-1) U=0$, где $D=U^{-1} \int_{-1}^{1} \mu^{2} J d \mu-$ коэффициент квазидиффузии. Если подставить в это уравнение диффузионное решение $U=$ const, то получим ОДУ для коэффициента квазидиффузии $\frac{\partial D}{\partial r}=\frac{\eta}{2 r}(1-3 D)$. Например, в плоской геометрии отсюда получаем $D=$ const или $\int_{-1}^{1} \mu^{2} J d \mu=$ const, что не следует из кинетического уравнения и не является его решением. 
В общем случае уравнение для нахождения плотности в разных приближениях отличается от кинетической модели на величину

$$
\delta=\frac{1}{r^{\eta}} \frac{\partial}{\partial r}\left(r^{\eta} D U\right)+\frac{\eta}{2 r}(D-1) U-\frac{1}{3} \frac{\partial U}{\partial r} .
$$

Видно, что эта величина даже в плоской геометрии

$$
\delta=\frac{\partial}{\partial r}\left[\left(D-\frac{1}{3}\right) U\right]=\frac{\partial}{\partial r} \int_{-1}^{1}\left(\mu^{2}-\frac{1}{3}\right) J d \mu
$$

может быть большой из-за неограниченности производных интенсивности по пространству. Поэтому в вакууме решения уравнений (21) и (23) могут сильно различаться. Хотя коэффициент квазидиффузии меняется в ограниченном диапазоне $[0,1]$, интенсивность и производные $\partial J / \partial r$ и $\partial U / \partial r$ меняются неограниченно, а потому в вакууме (в общем случае в оптически прозрачных средах) решения УП в Д, $\mathrm{P}_{1}$ и $\mathrm{P}_{1 / 3}$, приближениях могут как угодно сильно отличаться от решения кинетического уравнения.

Возникает вопрос, а можно ли изменить уравнение (23) так, чтобы получить решение в Д, $\mathrm{P}_{1}$ и $\mathrm{P}_{1 / 3}$ приближениях, совпадающее с решением кинетического уравнения (21)?

Оказывается можно, если в уравнение (23) ввести дополнительное слагаемое $\alpha_{k} S$, где $\alpha_{k}$ - согласованный с кинетическим решением коэффициент, который будет обеспечивать выполнение условий равенства решений уравнения (23) и уравнения (21): $U_{d i f}=U_{k i n}=\int_{-1}^{1} J d \mu$. Здесь введены индексы dif и kin, чтобы отличать решение диффузионного уравнения (23) от решения кинетического уравнения (21).

Равенство потоков в вакууме выполняется автоматически и вытекает из одинакового во всех приближениях уравнения для потока: $\left(\partial r^{\eta} S\right) / \partial r=0$. Решение этого уравнения $r^{\eta} S=$ const при совпадающих граничных условиях $S_{d i f}\left(r_{0}\right)=S_{k i n}\left(r_{0}\right)$, где $S_{k i n}\left(r_{0}\right)=\int_{-1}^{1} \mu J\left(r_{0}\right) d \mu-$ граничный поток, вычисленный из кинетического решения в точке $r_{0}$, дает равенство потоков во всей рассматриваемой системе $S_{d i f}=S_{k i n}$.

Введение дополнительного слагаемого $\alpha_{k} S$ было использовано в работе [12] для учета кинетических эффектов при решении диффузионных уравнений. Согласованный с кинетическим решением пробег в этой работе назван кинетическим пробегом и вычислялся в общем случае по формуле

$$
l_{k}^{-1}=S_{k i n}^{-1}\left(\frac{1}{c} \frac{\partial S_{k i n}}{\partial t}+\frac{\partial}{3 \partial r}\left[(3 D-1) U_{k i n}\right]+\frac{\eta}{2 r}(3 D-1) U_{k i n}\right) .
$$


Основным недостатком этой формулы является то, что она может давать отрицательные значения пробега.

При введении дополнительного слагаемого $\alpha_{k} S$ уравнение (23) в вакууме приобретает вид

$$
\frac{1}{3} \frac{\partial U_{d i f}}{\partial r}+\alpha_{k} S_{d i f}=0
$$

где $\alpha_{k}=-\frac{1}{3 S_{k i n}} \frac{\partial U_{k i n}}{\partial r}$.

В уравнении (24) естественно потребовать положительность согласованного коэффициента для корректности диффузионного уравнения, так как возможны ситуации, когда знаки потока и производной плотности излучения совпадают, что приведёт к отрицательности кинетического пробега.

Подставляя $\alpha_{k}$ в уравнение (24), получаем равенство

$$
S_{k i n} \frac{\partial U_{d i f}}{\partial r}=S_{d i f} \frac{\partial U_{k i n}}{\partial r} .
$$

При совпадении потоков на границе $S_{d i f}\left(r_{0}\right)=S_{k i n}\left(r_{0}\right)$ с учетом соотношения (20) получаем $S_{d i f}=S_{k i n}$. Тогда из (25) следует $\partial U_{d i f} / \partial r=\partial U_{k i n} / \partial r$. Это равенство гарантирует выполнение соотношения $U_{d i f}=U_{k i n}$ при совпадающих граничных условиях $U_{d i f}\left(r_{0}\right)=U_{k i n}\left(r_{0}\right)$.

Таким образом, для получения эквивалентных решений, из кинетического уравнения на границе можно сформировать граничное условие для потока в (22), а для уравнения (23) - граничное условие для плотности.

В результате вышеприведенных рассуждений можно сформулировать лемму.

Лемма. Для того чтобы решения в Д, $P_{1}$ и $P_{1 / 3}$ приближениях совпадали с решением кинетического уравнения в вакууме, необходимо введение положительного коэффициента $\alpha_{k}$ и согласованных граничных условий $U_{\text {dif }}\left(r_{0}\right)=U_{k i n}\left(r_{0}\right), S_{d i f}\left(r_{0}\right)=S_{k i n}\left(r_{0}\right)$.

Очевидно, что в вакууме Д, $\mathrm{P}_{1}$ и $\mathrm{P}_{1 / 3}$ приближения при введении коэффициента $\alpha_{k}$ переходят в КП приближение. Поэтому для КП приближения на основе леммы можно сформулировать теорему эквивалентности.

Теорема эквивалентности КП приближения (в вакууме).

Решение КП приближения будет совпадать с решением кинетического уравнения в вакууме при положительном коэффициенте $\alpha_{k}$ и согласованных условиях на границе $U_{\text {dif }}\left(r_{0}\right)=U_{k i n}\left(r_{0}\right), S_{\text {dif }}\left(r_{0}\right)=S_{k i n}\left(r_{0}\right)$. 
Если бы решение в КП приближении совпадало с решением кинетического уравнения, то этот подход являлся бы точным методом для уравнения переноса. Однако, из-за возможных нарушений положительности коэффициента $\alpha_{k}$ КП приближение является приближенным методом решения кинетического уравнения.

\section{4. Сравнение решений нестационарного уравнения переноса в различ- ных приближениях в оптически прозрачной среде}

В общем нестационарном случае введение согласованного с кинетическим решением системы (1) коэффициента $\alpha_{k}$ приводит к уравнениям

$$
\begin{aligned}
& \frac{1}{c} \frac{\partial U_{v}}{\partial t}+\frac{1}{r^{\eta}} \frac{\partial\left(r^{\eta} S_{v}\right)}{\partial r}+\alpha_{c v} U_{v}=\alpha_{c v} B_{v}, \\
& \underline{\beta} \frac{\partial S_{v}}{\partial t}+\frac{1}{3} \frac{\partial U_{v}}{\partial r}+\left(\alpha_{s v}+\alpha_{c v}+\alpha_{k v}\right) S_{v}=0,
\end{aligned}
$$

где коэффициент $\alpha_{k v}=S_{v}^{-1}\left[\frac{(1-\beta)}{c} \frac{\partial S_{v}}{\partial t}+\frac{\partial}{\partial r}\left(D_{v}-\frac{1}{3}\right) U_{v}+\frac{\eta}{2 r}\left(3 D_{v}-1\right) U_{v}\right]$. Коэффициент $\alpha_{k}$ имеет размерность коэффициентов поглощения и рассеяния.

Во втором варианте КП приближения, предложенном в [12], вместо согласованного коэффициента $\alpha_{k}$ вводится безразмерный коэффициент $m_{v}$, который приводит к системе

$$
\begin{aligned}
& \frac{1}{c} \frac{\partial U_{v}}{\partial t}+\frac{1}{r^{\eta}} \frac{\partial\left(r^{\eta} S_{v}\right)}{\partial r}+\alpha_{c v} U_{v}=\alpha_{c v} B_{v}, \\
& m_{v} \frac{\partial U_{v}}{\partial r}+3 \alpha_{v} S_{v}=0
\end{aligned}
$$

где $m_{v}=\left(S_{k i n} / S_{d i f}\right)_{v}-$ коэффициент КП, $S_{v, k i n}=\int_{-1}^{1} \mu J_{v} d \mu-$ кинетический поток, $S_{v, d i f}=-\frac{1}{3 \alpha_{v}} \frac{\partial}{\partial r}\left(\int_{-1}^{1} J_{v} d \mu\right)-$ аналог диффузионного потока, вычисленный по интенсивности или плотности излучения из кинетического уравнения.

Естественно при решении системы (27) потребовать положительности коэффициента КП, так как возможны ситуации, когда знаки диффузионного и кинетического потоков излучения не совпадают. Теоретически знаки диффузионного и кинетического потоков могут не совпадать, так как нет эквивалентности между решениями гиперболических и диффузионных уравнений. По этой же причине диффузионный и кинетический потоки могут не совпадать и по абсолютной величине. Но, исходя из физического смысла, 
это не должно быть бесконечно большой величиной. Поэтому для корректности задачи и избежания нефизических явлений при численной реализации на коэффициент КП накладываются естественные ограничения $0<m_{\min } \leq$ $\leq m_{\mathrm{v}} \leq m_{\max }$.

Заметим, что если потребовать выполнение равенства

$$
\frac{1}{3} \frac{\partial U_{d i f}}{\partial r}+\alpha_{k} S_{d i f}=\frac{1}{3} \frac{\partial U_{k i n}}{\partial r}+\alpha_{k} S_{k i n}
$$

то коэффициент $\alpha_{k}$ можно выбирать произвольным положительным числом. Положительность нужна только для корректного решения уравнения диффузии. Формально из уравнения (28) получаем новую формулу для $\alpha_{k}$ : $\alpha_{k}=\frac{1}{3\left(S_{d i f}-S_{k i n}\right)} \frac{\partial\left(U_{k i n}-U_{d i f}\right)}{\partial r}$, но использовать её сложно из-за возможных возникновений неопределенности 0/0 при $U_{d i f} \rightarrow U_{k i n}, S_{d i f} \rightarrow S_{k i n}$. Однако выражение (28) позволяет в случае получения отрицательных $\alpha_{k}$ в формуле (24) решать уравнение диффузии с ненулевой правой частью $f=\frac{1}{3} \frac{\partial U_{k i n}}{\partial r}+\alpha_{k} S_{k i n}$ и любым положительным $\alpha_{k}$, например, $\alpha_{k}=1$ в вакууме или $\alpha_{k}=\alpha_{s}+\alpha_{c}$ в общем случае.

$\mathrm{B}$ переносе нейтронов в последнее время широкое распространение получил крупносеточный конечно-разностный метод CMFD (coarse-mesh finite difference) [35], в котором используется процедура эквивалентности типа (28) диффузионного и кинетического уравнений с коэффициентом $\alpha_{k}=1$. Для обеспечения эквивалентности вводится двухэтапный итерационный процесс. На первом этапе из кинетического уравнения вычисляются $U_{k i n}^{v+1 / 2}, S_{k i n}^{v+1 / 2}$, на втором этапе из диффузионного уравнения - $U_{d i f}^{v+1}, S_{d i f}^{v+1}$, где $v$ - итерационный индекс. Для получения диффузионного уравнения в методе CMFD применяется следующая итерационная процедура:

$$
\frac{1}{3} \frac{\partial U_{d i f}^{v+1}}{\partial r}+S_{d i f}^{v+1}=\left(\frac{1}{3} \frac{\partial U_{k i n}^{v+1 / 2}}{\partial r}+S_{k i n}^{v+1 / 2}\right) \frac{U_{d i f}^{v+1}}{U_{k i n}^{v+1 / 2}}
$$

которая приводит к решению уравнения диффузии (27) с уравнением для потока вида $S_{d i f}^{v+1}=-\left(\frac{1}{3} \frac{\partial}{\partial r}-D_{k i n}^{v+1 / 2}\right) U_{d i f}^{v+1}$ и корректировочным коэффициентом $D_{k i n}^{v+1 / 2}=\left(\frac{1}{3} \frac{\partial U_{k i n}^{v+1 / 2}}{\partial r}+S_{k i n}^{v+1 / 2}\right) / U_{k i n}^{v+1 / 2}$. При сходимости итераций по- 
лучаем $U_{d i f}^{v+1}=U_{k i n}^{v+1 / 2}, S_{d i f}^{v+1}=S_{k i n}^{v+1 / 2}$ и, как следствие, равенство (28). Однако появление в полученном уравнении

$$
\frac{1}{c} \frac{\partial U_{v}}{\partial t}-\frac{1}{3 r^{\eta}} \frac{\partial}{\partial r}\left(r^{\eta} \frac{\partial U_{d i f}^{v+1}}{\partial r}\right)+\frac{1}{r^{\eta}} \frac{\partial}{\partial r}\left(r^{\eta} D_{k i n}^{v+1 / 2} U_{d i f}^{v+1}\right)+\alpha_{c v} U_{v}=\alpha_{c v} B_{v}
$$

дополнительного члена $\frac{1}{r^{\eta}} \frac{\partial}{\partial r}\left(r^{\eta} D_{k i n}^{v+1 / 2} U_{d i f}^{v+1}\right)$ может усложнить решение уравнения диффузии и привести к неустойчивости [36].

Фактически при положительном коэффициенте $m$ приближение КП совпадает с кинетическим уравнением, так как первое уравнение системы (27) является проинтегрированным уравнением переноса, а второе уравнение системы (27) при $S_{v, d i f}=-\frac{1}{3 \alpha_{v}} \frac{\partial U_{v}}{\partial r}$ является кинетическим потоком, записанным в параболической форме

$$
S_{v}=-\frac{m_{v}}{3 \alpha_{v}} \frac{\partial U_{v}}{\partial r}=-\frac{S_{v, k i n}}{S_{v, \text { dif }}} \frac{1}{3 \alpha_{v}} \frac{\partial U_{v}}{\partial r}=S_{v, k i n} .
$$

Поэтому из способа построения КП приближения следует справедливость леммы и теоремы эквивалентности в общем случае. Эквивалентность для КП приближения как в оптически прозрачных, так и в оптически плотных средах выполняется при положительности коэффициентов $\alpha_{k}>0$ или $m>0$ и выполнении на границе условий $U_{d i f}\left(r_{0}\right)=U_{k i n}\left(r_{0}\right)$ и $S_{d i f}\left(r_{0}\right)=S_{k i n}\left(r_{0}\right)$.

Эквивалентность решений кинетического уравнения и КД приближения доказана в [11].

\section{Заключение}

В работе рассмотрено теоретическое сравнение различных приближений системы уравнений переноса теплового излучения в оптически плотных и прозрачных средах. В результате проведенных исследований можно сказать, что в оптически плотных средах решения в рассмотренных приближениях стремятся к решению кинетического уравнения при увеличении оптической толщины. Аналитически получено, что коэффициент $m_{v} \rightarrow 1$, а коэффициент $D_{v} \rightarrow 1 / 3$. Это следует из асимптотического анализа. В оптически прозрачных средах совпадение с решением кинетического уравнения возможно только в приближениях квазипереноса или квазидиффузии.

В оптически прозрачных средах в Д, $\mathrm{P}_{1}$ и $\mathrm{P}_{1 / 3}$ приближениях надо вводить дополнительные члены или корректирующие множители, приводящие 
к КД или КП приближениям, для вычисления которых необходимо использовать решение кинетического уравнения. Этот способ позволяет получать решения в более простых Д, $\mathrm{P}_{1}$ и $\mathrm{P}_{1 / 3}$ приближениях, максимально приближенные к решению кинетического уравнения.

Однако при использовании КД или КП приближений есть существенная разница. КД приближение приводит к гиперболической системе, для которой сложно построить монотонную схему повышенного (выше первого) порядка точности. КП приближение приводит к параболической системе, для которой можно построить монотонную схему второго порядка точности.

\section{СПИСОК ЛИТЕРАТУРЫ}

1. Д. Белл, С. Глесстон. Теория ядерных реакторов. - М.: Атомиздат, 1974, 494 с.;

G. Bell, S. Glasstone. Nuclear Reactor Theory. Reinhold Company, 1970, $494 \mathrm{p}$.

2. В.В. Смелов. Лекции по теории переноса нейтронов. - М.: Атомиздат, 1978, 216 с.;

V.V. Smelov. Lekzii po teorii perenosa neitronov. - M.: Atomizdat, 1978, $216 \mathrm{~s}$.

3. Я.Б. Зельдович, Ю.П. Райзер. Физика ударных волн и высокотемпературных гидродинамических явлений. - М.: Наука, 1966, 688 с.;

Ia.B. Zeldovich, Iu.P. Raizer. Fizika udarnikh voln i visokotemperaturnikh iavlenii. - M.: Nauka, 1966, $688 \mathrm{~s}$.

4. А.Н. Тихонов, А.А. Самарский. Уравнения математической физики. - М.: Наука, $1966,724 \mathrm{c}$;

A.N. Tikhonov, A.A. Samarskii. Uravneniia matematicheskoi fiziki. - M.: Nauka, 1966, 724s.

5. Ryan McClarren, James Paul Holloway, Thomas Brunner. On solution to the Pn equations for thermal radiative transfer // J. Comp. Phys., 2008, v.227, p.2864-2885.

6. Patrick S. Brantley, Edward W. Larsen. The simplified P3 approximation// Nuclear science and engineering: 2000, v.134, p.1-21.

7. Р.М. Шагалиев и др. Методика САТУРН-2005. Математические модели, алгоритмы и программы решения многомерных задач переноса частиц и энергии // ВАНТ, 2013, в.4, c. 17-30;

R.M. Shagaliev i $d r$. Metodika SATURN-2005. Matematicheskie modeli, algoritmi I programmi // VANT, 2013, v.4, s.17-30.

8. А.В. Бабанов и др. Методика МИМОЗА-НДЗД. Расчет трехмерных задач спектрального переноса излучения // ВАНТ, 2012, в.2, с.64-72;

A.V. Babanov $i d r$. Metodika MIMOZA-ND3D. Raschet trekhmernikh programm // VANT, 2012, v.2, s.64-72.

9. В.Ю. Гусев, М.Ю. Козманов, Е.Б. Рачилов. Метод решения неявных разностных уравнений, аппроксимирующих системы уравнений переноса и диффузии излучения // ЖВМ и МФ, 1984, т.24, N12, с.1842-1849;

V.Ju. Gusev, M.Ju. Kozmanov, E.B. Rachilov. A method of solving implicit difference equations approximating systems of radiation transport and diffusion equations // USSR Comp. Math. Math. Phys., 1984, v.24:6, p.156-161. 
10. А.Д. Гаджиев, А.А. Шестаков. Метод «Ромб» для решения многогруппового уравнения переноса излучения в Р1-приближении // ВАНТ, 1989, в.3, с.66-70;

A.D. Gadzhiev, A.A. Shestakov. Metod «Romb» dlja reshenija mnogogruppovogo uravnenija perenosa izluchenija v P1-priblizhenii // VANT, 1989, v.3, s.66-70.

11. В.Я. Гольдин. Квазидиффузионный метод решения кинетического уравнения // ЖВМ и МФ, 1964, т.4, №6, С.1078-1087;

V.Ya. Gol'din. A quasi-diffusion method of solving the kinetic equation // USSR Comp. Math. Math. Phys., 1964, v.4:6, p.136-149.

12. W.A. Wiesequist, D.Y. Anistratov, J.E. Morel. A cell-local finite difference discretization of the low order of the quasidiffusion equations for neutral particle transport on unstructured quadrilateral meshes // J. Comp. Phys., 2014, v.273, p.343357.

13. E.N. Aristova. Simulation of radiation transport in channel on the basis of quasidiffusion method // Transport Theory and Statistical Physics, 2008, v.37 (05-07), p.483-503.

14. L. Chacon, G. Chen, D.A. Knoll, C. Newman, H. Park et al. Multiscale high- order/loworder (HOLO) algorithms and applications // J. Comp. Phys., Feb. 2017, v.330, p.21-45.

15. Н.Г. Карльханов, М.Ю. Козманов. Учет кинетических эффектов в диффузионном приближении для расчета переноса излучения // ВАНТ, 2010, в.4, с.3-9;

N.G. Karlikhanov, M.Ju. Kozmanov. Uchet kineticheskikh effektov v diffuzionnom priblizhenii dlja rascheta perenosa izluchenija // VANT, 2010, v.4, s.3-9.

16. Д.А. Кошутин, А.А. Шестаков. Решение двумерного уравнения переноса теплового излучения в приближении многогруппового квазипереноса // ВАНТ, 2017, в.3, с.39-50; D.A. Koshutin, A.A. Shestakov. Reshenie dvumernogo uravnenija perenosa teplovogo izluchenija v priblizhenii mnogogruppovogo kvaziperenosa // VANT, 2017, v.3, s.39-50.

17. Д.А. Кошутин, А.А. Шестаков. Приближенный метод решения уравнения переноса теплового излучения // Тезисы докладов Международного семинара 'Супервычисления и матмоделирование', Саров, 2018, с.73;

D.A. Koshutin, A.A. Shestakov. Priblizhennii metod reshenija uravnenija perenosa teplovogo izluchenija // Tezisi 'Supervichislenija i matmodelirovanie', Sarov, 2018, s.73.

18. А.А. Шестаков. Безусловно устойчивые разностные схемы для решения задачи переноса лучистой энергии в диффузионном и Р1-приближениях // ВАНТ, 1993, в.3, с.47-53;

A.A. Shestakov. Bezuslovno ustoichivie raznostnie skhemi dlja reshenija zadachi perenosa luchistoi energii v diffuzionnom i P1-priblizhenijakh // VANT, 1993, v.3, s.47-53.

19. А.Д. Гаджиев, А.А. Шестаков. Двумерная методика «Ромб» для численного решения уравнений переноса излучения в многогрупповом Р1-приближении // ВАНТ, 1990, в.1, c.41-47;

A.D. Gadzhiev, A.A. Shestakov. Dvumernaja metodika «Romb» dlja chislennogo reshenija uravnenii perenosa izluchenija v mnogogruppovom P1-priblizhenii // VANT, 1990, v.1, s.41-47.

20. А.В. Уракова, И.С. Чубарешко, А.А. Шестаков. Решение системы уравнений переноса теплового излучения в различных приближениях // Тезисы докладов Международного семинара 'Супервычисления и матмоделирование', Саров, 2018, с.75;

A.V.Urakova, I.S. Chuboreshko, A.A. Shestakov. Reshenie sistemi uravnenii perenosa teplovogo izluchenija v razlichnikh priblizhenijakh // Tezisi 'Supervichislenija i matmodelirovanie', Sarov, 2018, s.75.

21. А.Д. Гаджиев, А.А. Шестаков, В.Н. Селезнев, Е.М. Романова. Методика ТОМ4-КД 
для математического моделирования двумерных уравнений переноса излучения в многогрупповом квазидиффузионном приближении // ВАНТ, 2001, в.4, с.48-59;

A.D. Gadzhiev, A.A. Shestakov, V.N. Seleznev, E.M. Romanova. Metodika TOM4-KD dlja chislennogo modelirovanija dvumernikh uravnenii perenosa izluchenija v mnogogruppovom kvazidiffuzionnom priblizhenii // VANT, 2001, v.4, s.48-59.

22. А.Д. Гаджиев, А.А. Шестаков, С.А. Грабовенская, В.В. Завьялов. Применение TVD подхода к решению уравнения переноса теплового излучения квазидиффузионным методом // ВАНТ, 2010, в.3, с.3-14;

A.D. Gadzhiev, A.A. Shestakov, S.A. Grabovenskaja, V.V. Zavjalov. Primenenie TVD podkhoda $\mathrm{k}$ resheniju uravnenija perenosa teplovogo izluchenija kvazidiffuzionnim metodom // VANT, 2010, v.3, s.3-14.

23. С.А. Грабовенская, А.А. Шестаков. Анализ некоторых схем для решения уравнения переноса излучения квазидиффузионным методом // ВАНТ, 2011, в.4, с.3-15;

S.A. Grabovenskaja, A.A. Shestakov. Analiz nekotorikh skhem dlja reshenija uravnenija perenosa izluchenija kvazidiffuzionnim metodom // VANT, 2011, v.4, s.3-15.

24. С.А. Грабовенская, В.В. Завьялов, А.А. Шестаков. Конечно-объемная схема ГРОМ для решения переноса излучения квазидиффузионным методом // ВАНТ, 2014, в.3, с. 47-58; S.A. Grabovenskaja, V.V. Zavjalov, A.A. Shestakov. Konechno-obemnaja schema GROM dlja reshenija perenosa izluchenija kvazidiffuzionnim metodom // VANT, 2014, v.3, s.47-58.

25. Г.И. Марчук, В.И. Лебедев. Численные методы в теории переноса нейтронов. - М.: Атомиздат, 1981, 454 с.;

G.I. Marchuk, V.I. Lebedev. Chislennie metodi v teorii perenosa neitronov. - M.: Atomizdat, $1981,454 \mathrm{~s}$.

26. E.W. Larsen. Unconditionally Stable Diffusion Acceleration of the Transport Equation // Transport Theory Statist. Phys., 1982, v.11, p.29.

27. А.Д. Гаджиев, И.А. Кондаков, А.А. Шестаков. RDSA-метод для численного решения уравнения переноса нейтронов // ВАНТ, 2007, в.2, с.3-19;

A.D. Gadzhiev, I.A. Kondakov, A.A. Shestakov. RDSA-metod dlja chislennogo reshenija uravnenija perenosa neitronov // VANT, 2007, v.2, s.3-19.

28. А.Д. Гаджиев, Д.А. Кошутин, А.А. Шестаков. Метод дискретных ординат с TVD реконструкцией и синтетический метод ускорения итераций для численного решения уравнения переноса теплового излучения // ВАНТ, 2013, в.3, с.3-15;

A.D. Gadzhiev, D.A. Koshutin, A.A. Shestakov. Metod diskretnikh ordinat s TVD - rekonstrukziei i sinteticheskii metod uskorenija iterazii dlja chislennogo reshenija uravnenija perenosa teplovogo izluchenija // VANT, 2013, v.3, s.3-15.

29. А.Д. Гаджиев, Д.А. Кошутин, А.А. Шестаков. DSn-метод с TVD-реконструкцией и синтетическим P1SA-методом ускорения итераций для численного решения двумерного уравнения переноса теплового излучения в осесимметричной RZ-геометрии // BAHT, 2016, в.4, с.3-19;

A.D. Gadzhiev, D.A. Koshutin, A.A. Shestakov. DSn-metod s TVD-rekonstrukziei i sinteticheskim P1SA-metodom uskorenija iterazii dlja chislennogo reshenija dvumernogo uravnenija perenosa teplovogo izluchenija v osesimmetrichnoi RZ-geometrii // VANT, 2016, v.4, s.3-19.

30. M.L. Adams, E.W. Larsen. Fast iterative methods for discrete-ordinates particle transport calculations // Progress in Nuclear Energy, 2002, v.40:1, p.3-159. 
31. М.Н. Оцисик. Сложный теплообмен. - М.: Мир, 1976, 616 с.;

M.N. Ozisik. Radiative transfer and interactions with conduction and convection. - New York, 1973, $616 \mathrm{p}$.

32. E.W. Larsen, G.C. Pomraning, V.C. Badham. Asymptotic analysis of radiative transfer problems // JQSRT, 1983, v.29, p.285.

33. R. McClarren, J. P. Holloway. Establishing an Asymptotic Diffusion Limit for Riemann Solvers on the Time-Dependent Pn Equations // Mathematics and Computation, Supercomputing, Reactor Physics and Nuclear and Biological Applications Palais des Papes, Avignon, France, 2005.

34. А.А. Шестаков. О диффузионных свойствах схемы Ромб для Р1 уравнений // ВАНТ, 2011, в.2, с.56-62;

A.A. Shestakov. O diffuzionnikh svoistvakh skhemi Romb dlja P1 uravnenii // VANT, 2011, v.2, s.56-62.

35. N.Z. Cho, G.S. Lee, C.J. Park. Partial Current-Bazed CMFD Acceleration of the 2D/1D Fusion Method for 3D Whole-Core Transport Calculations // Trans. American Nuclear Society, 2003, v.88, p.594.

36. А.А. Шестаков. Исследование возникновения осцилляций при решении одномерного уравнения миграции / Снежинск: РФЯЦ-ВНИИТФ, 1996, препринт №100, 25 с.;

A.A. Shestakov. Issledovanie bozniknovenija oszilljazii pri reshenii odnomernogo uravnenija migrazii / Snezhinsk: RFJAZ-VNIITF, 1996, preprint №100, 25 s.

Поступила в редакцию 18.07.2019

После доработки 02.12.2019

Принята к публикации 23.12.2019 